\title{
Depression in Clinical Practice: A Retrospective Case Record Analysis
}

\author{
Anil K Agarwal
}

\begin{abstract}
Aims and objectives: The aim of this study is to study the natural course of depression in a private clinic setting. Materials and methods: The records of all patients suffering from depression excluding bipolar and schizoaffective disorders diagnosed according to DSM IV were scrutinized from November 1, 2017, to October 31, 2018.

Observations: A total of 702 patients were diagnosed as suffering from depression. Nearly $13 \%$ had a change in diagnosis of bipolar disorder or obsessive-compulsive disorder (OCD) and other conditions. Nearly 80 patients' records were not adequate, and therefore, only 528 patients were taken for detailed study. All these patients were analyzed for various clinical details. Many patients suffering from depression require a change of diagnosis mainly to bipolar disorder and OCD. Depression is generally a chronic recurring illness, but the pattern of recurrences is variable. More men seek treatment, while depression is more common in females. Similarly, more young people seek treatment while depression is more common in the elderly. The number of chronic patients increased with age. Most patients came to the clinic only when they were feeling worse and discontinue treatment as soon as they get better. Resistant depression requires reevaluation of diagnosis and reassessment of psychosocial factors.

Keywords: Depression, Natural history, Psychiatric clinic.

Indian Journal of Private Psychiatry (2020): 10.5005/jp-journals-10067-0049
\end{abstract}

\section{INTRODUCTION}

Depression is a chronic recurrent disorder with profound effects on the patient's personal life, work performance, and health. ${ }^{1}$ National Mental Health Survey ${ }^{2}$ reports a prevalence of $2.7 \%$ for depressive disorders in India. This figure could be a lower estimate as most surveys miss depression in the general population as people do not consider depression as an illness. The Global Burden of Disease and Risk Factors study ${ }^{3}$ reports that the prevalence of depression in men is $1.9 \%$ and in women is $3.2 \%$. It is also estimated that depression will be the largest cause of disability from 2020. The overall prevalence rates vary from 1.7 to 77 per thousand. ${ }^{4}$ Follow-up studies reveal that roughly a third had no relapse., ${ }^{5,6}$ Diagnosis changes to bipolar disorder in77\%. ${ }^{7}$ Reviews of Indian research on different aspects are available..$^{8,9}$ The treatment gap is huge both in India and in the West. Response to medical treatment is at best equivocal. ${ }^{10}$ Residual symptoms are a rule in $80-90 \%$ of patients. ${ }^{11,12}$ The etiology of depression is evolving, and it is considered as the culmination of interaction between small-effect genes, environment, and biological systems. Epigenetics indicate that bereavement in childhood or other stresses may induce changes that are permanent and inheritable. There are not many studies of depression in its natural setting. The present study is an attempt to report the real-life situation as observed in a private psychiatric clinic in Northern India.

The present study analyses records of patients suffering from depression for one year. This is a retrospective analysis of patients seen in a private practice clinic in North India. The author had been practicing at the same place for the last fifty years. The author has recently started keeping records in the electronic format, while earlier the records were kept in handwritten files. These records are neither complete nor claim of any standardization. The records were kept for effective clinical work. The data from this clinic have already been published ${ }^{13}$ earlier for determining the patterns of patients
Department of Gl Surgery \& Liver Transplantation, King George's Medical College, Lucknow, Uttar Pradesh, India

Corresponding Author: Anil K Agarwal, King George's Medical College, Lucknow, Uttar Pradesh, India, Phone: +91 9415006128, e-mail:mradulanil@gmail.com

How to cite this article: Agarwal AK. Depression in Clinical Practice: A Retrospective Case Record Analysis. Ind J Priv Psychiatry 2020;14(1): $1-5$.

Source of support: Nil

Conflict of interest: None

coming to the clinic and depression constituted $40 \%$ of the clinic attendance. Another paper described the development ${ }^{14}$ of the clinic in the past twenty years. These publications were aimed at looking at psychiatric patients in totality. The current presentation is to look at the clinical presentation and treatment response in a natural setting in a busy private psychiatric clinic.

\section{Aims and Objectives}

The aim of this study is to study the natural course of depression in a private clinic setting.

\section{Materials and Methods}

The records of all patients suffering from depression excluding bipolar and schizoaffective disorders diagnosed according to DSM IV were scrutinized from November 1, 2017, to October 31, 2018. The diagnosis was generally best fit. The cases are being recorded in a locally made software that can analyze patients based on diagnosis on the first visit. The changes made in symptomology and treatment at later dates are recorded but do not replace the original 
diagnosis. This provides us an opportunity to reassess diagnosis during follow-up. No scales were used. The patients were rated for improvement, which was based on patients' reports and the clinician's observation, and three categories were used: no change/ worse, better, and well maintained (meant nearly normal). The case records of all these patients were checked for a revisit after one year. This is a busy clinic with 25-30 patients per day, and therefore, the patients needed to be examined quickly as is observed in most psychiatric outpatient clinics in India.

\section{Observations}

The analysis revealed that 702 patients were suffering from depression and major depressive disorders (MDD) during this period. The diagnosis was changed to bipolar affective disorder (BAD) in $81(11.5 \%)$ patients to obsessive-compulsive disorder (OCD) in $10(1 \%)$, and Schizophrenia and others in $3(0.4 \%)$. There was inadequate information in 80 records, and they were excluded. Finally, 528 patients were taken for final analysis. Further reevaluation one year later revealed that 2 more patients developed bipolar disorder and another 9 patients did not have proper data on outcome, and therefore, the outcome table has only 517 patients.

Age and sex distribution: Males constituted $60 \%$ of the sample while depression is said to be two times more in females. The sample has been divided into three age groups and the maximum number of cases is in the age group 25-50 years (Table 1).

\section{Age and Depression}

Depression occurs at every age. The onset of illness within six months of presentation in different age groups is $13 \%$. This indicates that fresh cases of depression occur all through life. No clear difference was observed between males and females. The number of patients in 6 months to 2 years and $2-5$ years remains around $20 \%$ each. Chronic depression tends to increase with age. Depression of 5 years or more increased to $50 \%$ in the $50+$ age group from $10 \%$ in below 25 years (Table 2).

\section{Clinical Presentation}

The common pattern observed is that of repeated relapses lasting for a few months with attacks occurring every few years. The longest interval was of 35 years between two attacks. Many

Table 1: Age and sex distribution

\begin{tabular}{lcc}
\hline Age & Male no. (\%) & Female no. (\%) \\
\hline $15-24$ & $33(11)$ & $24(11)$ \\
$25-50$ & $170(54)$ & $122(57)$ \\
$51+$ & $111(35)$ & $68(32)$ \\
Total & $314(60)$ & $214(40)$ \\
\hline
\end{tabular}

Table 2: Duration of illness and age

\begin{tabular}{lcccc}
\hline & $15-24$ & $25-50$ & $50+$ & \multicolumn{1}{l}{ Total } \\
Duration & No. (\%) & No. (\%) & No. (\%) & \multicolumn{1}{c}{ No. (\%) } \\
\hline 0-6 months & $16(28)$ & $39(13)$ & $15(8)$ & $70(13)$ \\
7 months-2 years & $20(34)$ & $71(24)$ & $25(14)$ & $116(22)$ \\
2-5 years & $16(28)$ & $71(24)$ & $49(28)$ & $136(26)$ \\
$>5$ years & $6(10)$ & $111(38)$ & $89(50)$ & $206(39)$ \\
Total & $58(11)$ & $292(55)$ & $178(34)$ & 528 \\
\hline
\end{tabular}

patients ignore milder attacks of depression and often lapses of recall may obliterate such history. There was a group of patients where illness became chronic after a few relapses. Many patients continue taking antidepressants for long periods without consultation as they feel better. Quite a few patients had seasonal relapses with relapse occurring at the time of change of season, that is, August to October and March to April. This observation appears specific to India and may be more in northern India where weather fluctuations are more pronounced. A few had worsening of symptoms during dark cloudy days of monsoon. Some had winter or summer depression. Most of the seasonal depression tends to convert to BAD. This being a chart review, many patients who had not come back or may have suffered a single attack are likely to be missed.

Symptomatology observed in this sample was of classic depression seen worldwide. Depressed mood, negative thinking, sleep disturbances, frequent crying, and guilt were observed. Psychomotor retardation was more frequent than agitation. The melancholic features were present in quite a few. The elderly tend to show mood-congruent delusions specifically of properties being taken away or stolen or fear of persecution from relatives and friends.

Family history: Nearly $20 \%$ of patients had a family history of depression in the first-degree relative. There were 108 patients out of 528 where a clear history of depression in first-degree relatives was observed. It must be emphasized that the majority of our patients are very reluctant in providing a family history of mental illness.

Treatment: These patients were generally treated with antidepressant medications, and tranquilizers were used when needed. Escitalopram was the first drug of choice, and the starting dose was 10-20 mg per day. Most patients react adversely to escitalopram in the early treatment, and therefore, it is combined with antianxiety drugs initially, which are later withdrawn. Patients having prolonged and severe depressions were prescribed escitalopram and bupropion in combination. The effort was to treat depression as fast as possible with the least number of side effects. Patients not responding to the above were shifted to venlafaxine. When tricyclics were used, the drug of choice was nortriptyline, and in very few cases amitriptyline or imipramine was used. Most patients who came early in illness responded to first-line treatment, i.e., escitalopram. This author believes that most patients with depression respond to a single selective serotonin reuptake inhibitor (SSRI), while others respond to combinations of antidepressants. All patients who do not adequately respond were carefully evaluated for OCD, and many were diagnosed with OCD. Other psychiatric illnesses, such as, schizophrenia, physical illnesses, and severe adverse circumstances were also carefully evaluated. Nonresponding patients were also screened for schizoaffective disorders. Once all of the above were excluded, very few patients were left in the category of resistant depression. Very few patients may need electroconvulsive therapy (ECT), but it can be a lifesaver in these patients. This author was not giving ECT in his clinic, and those require such treatment were referred to others but only six patients needed this treatment. But when needed, the ECT treatment was life-changing.

Anxiety is often a very disturbing symptom of some patients, and tranquilizers were used at an inadequate dose till such times the anxiety was controlled. But the antianxiety drugs were tapered 
Table 3: Age and outcome

\begin{tabular}{llllllll}
\hline Age & $0-25$ & $0-25$ & $26-50$ & $26-50$ & $50+$ & $50+$ & Total \\
\hline Outcome & Male No. (\%) & Female No. (\%) & Male No. (\%) & Female No. (\%) & Male No. (\%) & Female No. (\%) \\
Better & $10(36)$ & $10(42)$ & $68(40)$ & $53(44)$ & $47(42)$ & $27(44)$ & $215(42)$ \\
Maintained & $3(11)$ & $2(8)$ & $17(10)$ & $13(11)$ & $12(11)$ & $10(16)$ & $57(11)$ \\
Same or worse & $15(53)$ & $12(50)$ & $85(50)$ & $56(45)$ & $52(45)$ & $25(40)$ & $245(47)$ \\
Total & $28(5)$ & $24(5)$ & $170(33)$ & $122(24)$ & $111(21)$ & $62(12)$ & 517 \\
\hline
\end{tabular}

Male: 309

Female: 208

Table 4: Revisits after 1 year

\begin{tabular}{llllllrr}
\hline Age & $<25$ & $<25$ & $26-50$ & $26-50$ & $50+$ & $50+$ & Total \\
\hline Outcome & Male No. (\%) & Female No. (\%) & Male No. (\%) & Female No. (\%) & Male No. (\%) & Female No. (\%) & No.(\%) \\
Better & $7(70)$ & $2(20)$ & $29(39)$ & $31(38)$ & $9(22)$ & $5(20)$ & $83(34)$ \\
Maintained & 0 & $2(20)$ & $10(14)$ & $8(10)$ & $11(28)$ & $9(36)$ & $40(17)$ \\
Worse & $3(30)$ & $6(60)$ & $35(47)$ & $43(52)$ & $20(50)$ & $11(44)$ & $118(49)$ \\
Total & $10(4)$ & $10(4)$ & $74(20)$ & $82(34)$ & $40(17)$ & $25(10)$ & 241 \\
\hline
\end{tabular}

off as soon as the patient's depression and anxiety are reasonably controlled. Sleep is another problem that needs to be dealt with adequately. Most patients request a sleeping pill and feel that if they get good sleep, their depression will get better. Sleeping pills were given as required for as short a duration as possible. Very often there is a delayed sleep cycle where the patient sleeps late and wakes up late. The changed sleep cycle required cognitive behavior therapy for changing sleep timing. A changed sleep cycle is a common observation and many patients are unnecessarily given hypnotics when cognitive behavior therapy is the treatment of choice.

Outcome: This being a retrospective analysis of the case records, there were no specific criteria used for the outcome. The patient's last responses as recorded have been used as outcome measures. Therefore, only three categories were used: better, same/worse, well maintained. Well maintained were the patients who were euthymic for three months or more. This being a clinic-based study, one needs to remember that generally, patients visit the clinic when they are not feeling well. Nearly $42 \%$ were better, $11 \%$ well maintained, and $47 \%$ were the same or worse. Most of the patients in the same or worse group do improve with time. Quite a few patients continued taking antidepressants continuously for long periods after they got better. They come for consultation only when they felt the drugs were not working or drug availability had become an issue due to changes in drug availability laws (Tables 3 and 4).

Nearly $40 \%$ of males came for a revisit after one year (124 out of 309). On the other hand, $56 \%$ of females came for a revisit (117 out of 208). Females who revisited were more. The pattern of outcome was $34 \%$ better, $17 \%$ well maintained, and $49 \%$ the same or worse.

The reasons for dropout are recorded in Table 5. Most of these factors were combined in many patients. These were obtained from patients who came to the clinic after a long gap. Therefore, the numbers are small. Patients stop taking medicines as soon as they start feeling better, which often results in relapse. Sometimes associated illnesses were the cause of drug stoppage. There is a belief in the general population that treatment of two illnesses could be harmful, and many patients stop taking medicines if they have another illness. Two patients switched to mania. Many patients had multiple factors that contribute to dropout.
Table 5: Reasons for dropout

\begin{tabular}{lr}
\hline Stopped taking medicines & 43 \\
Persistent psychosocial problems & 18 \\
Physical diseases & 13 \\
Persistent agoraphobia and fear of closed space & 4 \\
Alcohol or drug abuse & 10 \\
Switched to mania_-bipolar & 2 \\
Sexual difficulties & 3 \\
\hline
\end{tabular}

\section{Discussion}

This study is a retrospective analysis of case records. The records were not standardized, nor were they complete in many aspects. Despite these deficiencies, this study provides certain specific observations about depression in our cultural setup. This study revealed that more males seek treatment in our country. Epidemiological data clearly reveals that depression is twice as common in females as in men. Ours is a male-dominated society, and males are breadwinners, so they get priority in treatment. The sample was divided into three categories according to age: below 25 years, $26-50$ years, and above 50 years. The proportion of males and females are almost similar in different age groups. More persons in the earning age group seek treatment, which reemphasizes the point that economics is an important factor in seeking treatment. Few elderlies seek treatment while depression is said to be more frequent in the elderly. These observations suggest that there could be a large mass of untreated depression. There is an urgent need to sensitize people regarding the treatability of depression as it affects the quality of life. The treatment gap in depression is estimated to be around $85.2 \%$ in India. The number of patients with long-duration continues to increase with age. Nearly $50 \%$ of patients in the 50 years plus group had long-term depression. This could be due to multiple factors. It could be due to residual symptoms, or the repeated depression may lead to chronicity and hence the need for early and effective treatment.

The majority of patients come for consultation only until they are depressed and discontinue as soon as they are better. This leads 
to repeated relapses. Many continue taking an antidepressant on their own as any reduction in dosage leads to discomfort. The discomfort may be due to serotonin withdrawal syndrome and not due to depression. There could also be withdrawal symptoms of minor tranquilizers if one were taking an additional benzodiazepine. The withdrawal symptoms happen more often when the patients are taking a combination of antidepressant and minor tranquilizers. This combination should be banned. The clinicians need to educate the patients and the families repeatedly about the need for longterm treatment. There is some evidence that continuous intake of antidepressants may lead to lowering of response. ${ }^{17}$

The clinical presentation revealed classical depressive symptomatology. This study did not reveal excessive somatization as recorded by many authors in the past. ${ }^{15-18}$ Is symptomatology changing? Many patients tend to get a relapse at the time of change of season, i.e., September/October and February/March. This could be more prevalent in North India where seasonal variation is larger. Some patients showed worsening of symptoms in the cloudy rainy season and not in winters.

The initial diagnosis of depression is often changed on follow-up. The diagnosis was changed to bipolar disorder in $10 \%$, to OCD in $1 \%$, and in very few to schizophrenia. Diagnostic change in depression to bipolar disorder is a function of the duration of follow-up and many more are likely to be revised to the diagnosis of bipolar disorder. Diagnostic changes in depression are in the range of $38-75 \%{ }^{5-7}$ Studies around the world stress the relationship between depression and OCD, but it has not been emphasized that some patients of OCD are wrongly diagnosed with depression and treated. Several patients with OCD do not reveal obsessive symptoms at the time of presentation and are sometimes diagnosed with depression.

The role of genetic factors in depression is estimated to be around $30-40 \%$ and the remaining variability is attributable to the environment. ${ }^{19}$ Genetic factors were observed in $20 \%$ in this study. The author believes that many more would have a positive family history if history is taken more carefully. Family history is an important element in treatment because it provides a definitive treatment plan. The possible reasons for not disclosing family history could be the fear that positive family history may mean a poor prognosis. A family history of illness also leads to a blame game. Many patients blamed their relatives as responsible for giving them this illness; on the other hand, some parents blamed themselves for giving this illness to their dear ones. To obtain good family history, following points need to be communicated to the relative. Positive family history does not mean that the illness is not treatable. The family history will provide us a possible course of the illness that will help us to treat the illness better. No one needs to be blamed for inducing the illness because genes and not one's bad deeds lead to mental illness. Positive family history helps the patients to differentiate between depression due to stress and depression without any substantial stress. Patients become more cooperative in drug treatment.

Treatment of depression had been very controversial. Some authors believe that drugs are no better than placebo. Inadequate response to treatment is emphasized by many. This is largely because the diagnosis of major depressive disorder includes many divergent disorders as the diagnosis is based on symptomatology and not on etiology. A recent article in Nature ${ }^{20}$ specifically emphasizes the inadequacy of the present diagnostic systems. This author believes that these poor response rates are since the diagnosis of major depressive disorder includes many diverse conditions due to which treatment response cannot be uniform. Proper evaluation of treatment response should start with distinguishing different conditions that could be included in this diagnosis. One must exclude bipolar depression, depression associated with $O C D$, depression due to prolonged persistent stresses, such as, losses, and chronic or life-threatening physical diseases. This author has observed that SSRIs and other antidepressants are highly effective if one excludes patients as described earlier and looks for symptoms suggestive of endogenous depression. The presence of psychomotor retardation is a good indicator of treatment response. There could be poor response in some cases where additional medicines may improve results.

Psychological help is an important factor in the treatment of depression. Some authors emphasize that the most crucial aspect of prescription is not what we prescribe but how we prescribe. ${ }^{21}$ There are interesting reports that one group of clinicians gets better results than other groups of clinicians because their therapeutic alliance is enabling. ${ }^{22}$ This author believes that the process of prescribing must be carefully planned specifically for each individual so that it takes care of his doubts and apprehensions.

Every patient of depression needs to be provided psychological help on the following lines. This is an illness that develops due to certain biological changes in the brain and is the result of multiple interactions the patient had with physical and social stresses. Any current stress could be a precipitating factor but is not the exclusive cause. Such individuals start evaluating all things negatively. There is a feeling of hopelessness and helplessness. Most patients of depression do improve despite the persisting cause. Patients need to be provided a feeling of hope and should know that it is a temporary phase and will change soon. Many patients do not want to take medicines as they feel that they should fight it out. They need to be told that they have already made efforts and have not succeeded. The medications will help them fight better. Many patients have such adverse circumstances that may perpetuate depression for long. They also need to be assured that they have already overcome some such events earlier. The twin facts need to be emphasized: it is temporary and there is always hope.

Many patients have suicidal thoughts, and these thoughts should not be ignored. Suicidal thoughts develop as the patient feels that they would not get better, that they are a burden on others, and there is a severe feeling of hopelessness and helplessness. They need to be told that the illness is eminently treatable. The feelings of hopelessness should be changed to feelings of hopefulness. One is often tempted to keep them in strict surveillance to avoid any further suicidal attempts. Patients often want to face challenges of the situation, but the family and the therapist are often afraid to take risk. There can be no hard and fast rule, but the patient's intention must be clearly understood; is he trying to force you in allowing him to escape so that he may commit suicide or he genuinely wants to make efforts to bolster his self-esteem. I often like to take risk and allow the patients to make efforts to mend their lives and it often succeeds. Patients with depression will have multiple psychosocial problems, such as, economic difficulties, job problems, and interpersonal conflicts. All of them need to be resolved by counseling.

Many of us are prescribing combinations of antidepressants with tranquilizers. This is a bad practice on two counts. When one needs to increase either antidepressant or tranquilizer, one unnecessarily increases the other. Secondly, continuous use will lead to habituation. Patients suffer double withdrawal symptoms of a tranquilizer and an SSRI. One can use them separately and taper tranquilizers first. 
Side effects: Many antidepressants induce disturbing side-effects. The commonest of them is weight gain. Avoid weight gain by using weight-neutral medications, but if they do not help, then use medications with weight control measures. Sexual difficulties are often increased by antidepressants and often patients refuse to take medicines due to these side effects. Use medicines with fewer sexual side effects, such as bupropion, mirtazapine, and vortioxetine. Other side-effects, such as constipation, drowsiness, and postural hypotension can be easily controlled. Long-term use can lead to the serotonergic syndrome.

\section{Conclusions}

Depression constitutes a major part of clinical practice. Depression can be an overt feature of many different diseases, such as OCD, schizophrenia, organic brain diseases, bipolar disorder, and schizoaffective disorders, and one should carefully rule them out. More males in the earning age group seek treatment. Most patients with depression discontinue treatment as soon as they get better, which results in relapse. Seasonal variation occurs in the months of September/October and February/March. Few patients show depression in dark cloudy months of the rainy season. Depression occurs at all ages. Treatment of depression is mainly dependent on antidepressants with psychosocial support and most patients respond well. Patients have multiple psychological problems and those need to be addressed psychologically. There is no rationale for using a fixed-dosage combination of antidepressants and benzodiazepines.

\section{Clinical Importance of the Study}

Depression constitutes a major part of clinical work in psychiatric practice. The diagnosis of depression includes many divergent disorders due to which treatment response appears equivocal. But if one teases out depressions with family history and endogenous quality, then the response is excellent. Most patients of depression tend to discontinue treatment as soon as they start feeling better, which leads to relapse. We must repeatedly emphasize to the patients and family members the need for prolonged treatment. Psychological issues need to be effectively managed for proper treatment. Treated patients of depression are our goodwill ambassadors and we must take good care.

Limitations: This is a retrospective analysis of one clinician's records; therefore, it is likely to be biased in many ways. All clinicians tend to attract a set of clients. Clients who dropped out are missed. A single clinician has his own peculiar biases.

\section{References}

1. Kessler RC. The costs of depression. Psychiatr Clin North Am 2012;35(1):1-14. DOI: 10.1016/j.psc.2011.11.005.
2. Gururaj G, Varghese M, Benegal V, et al. Summary National Mental Health Survey of India, 2015-16. Bengaluru: National Institute of Mental Health and Neurosciences; 2016. NIMHANS Publication No. 128.

3. Lopez AD, Mathers CD, Ezzati M, et al. Global Burden of Disease and Risk Factors. Washington: The World Bank; 2006.

4. Reddy MV, Chandrashekhar CR. Prevalence of mental and behavioral disorders in India: a meta-analysis. Indian J Psychiatry 1998;40(2): 149-157.

5. Rao AV, Nammalvar N. The course and outcome in depressive illness. A follow-up study of 122 cases in Madurai, India. Br J Psychiatry 1977;130:392-396. DOI: 10.1192/bjp.130.4.392.

6. Gada M. The course of depressive illness: a follow-up investigation of 92 cases. Indian J Psychiatry 1989;31(3):196-200.

7. Brown AS, Varma VK, MalhotraS, et al. Course of acute affective disorders in a developing country setting. J Nerv Ment Dis 1998;186(4):207-213. DOI: 10.1097/00005053-199804000-00002.

8. Grover S, Dutt A, Avasthi A. An overview of Indian research in depression. Indian J Psychiatry 2010;52(Suppl 1): 178-188. DOI: 10.4103/0019-5545.69231.

9. Sarkar S, Grover S. A sytematic review and meta-analysis of trials of treatment of depression from India. Indian J Psychiatry 2014;56(1):2938. DOI: 10.4103/0019-5545.124711.

10. Berlim MT, Turecki G. Definition, assessment, and staging of treatment-resistant refractory major depression: a review of current concepts and methods. Can J Psychiatry 2007;52(1):46-54. DOI: 10.1177/070674370705200108.

11. Fava GA, Tomba E. New modalities of assessment and treatment planning in depression: the sequential approach. CNS Drugs 2010;24(6):453-465. DOI: 10.2165/11531580-000000000-00000.

12. Fava GA, Tomba E, Grandi S. The road to recovery from depression: don't drive today with yesterday's map. Psychother Psychosom 2007;76(5):260-265. DOI: 10.1159/000104701.

13. Agarwal AK. Analysis of patients attending a private psychiatric clinic Indian J Psychiatry 2012;54(4):356-358. DOI: 10.4103/0019-5545.104824.

14. Agarwal AK. Psychiatric practice: over the years. Indian J Private Psychiatry 2016;10:10-15.

15. Gupta R, Khandelwal S, Varma VK, et al. Depressive symptoms: an intercentre comparison. Indian J Psychiatry 1982;24(4):380-382.

16. Derasari S, Shah VD. Comparison of symptomatology of depression between India and U.S.A. Indian J Psychiatry 1988;30(2):129-134.

17. Chaturvedi SK, Upadhyaya M. Double depression and somatization. Am J Psychiatry 1989;146(10):1354-1355. DOI: 10.1176/ajp.146. 10.1354b.

18. Teja JS, Narang RL, Aggarwal AK. Depression across cultures. British J Psychiatry 1971;119(550):253-260. DOI: https://doi.org/10.1192/ bjp.119.550.253.

19. Stotland NL. Recovery from depression. Psychiatry Clin North Am 2012;35(1):37-49. DOI: 10.1016/j.psc.2011.11.007.

20. Marshall $M$. The hidden links between mental disorders. Nature 2020;581(7806):19-21. DOI: 10.1038/d41586-020-00922-8.

21. Mintz DL, Flynn DF. How (not what) to prescribe: nonpharmacologic aspects of psychopharmacology. Psychiatr Clin North Am 2012;35:143-163. DOI: 10.1016/j.psc.2011.11.009.

22. E McKay KM, Imel ZE, Wampold BE. Psychiatrist effects in the psychopharmacological treatment of depression. J Affect Disord 2006;92(2-3):287-290. DOI: 10.1016/j.jad.2006.01.020. 